\section{Kurzer Fragebogen hilft bei der Diagnose COPD}

Martinez FJ et al. A new approach for identifying patients with undiagnosed chronic obstructive pulmonary disease. Am J Respir Crit Care Med 2017; 195: $748-756$

Die chronisch obstruktive Lungenerkrankung (COPD) wird ambulant häufig unterdiagnostiziert: Meist fällt sie erst durch eine Exazerbation oder eine deutliche Verschlechterung der Lungenfunktion auf. F. J. Martinez und Kollegen entwickelten nun einen Fragebogen, der in Kombination mit einer Peak-Flow-Messung in der Lage ist, Patienten zu identifizieren, die von einer Therapie profitieren würden.

Die Autoren führten hierzu zunächst eine prospektive Fall-Kontroll-Studie durch, für die aus dem ambulanten Bereich Patienten rekrutiert wurden. Als Fälle dienten dabei Patienten mit einer COPD und mindestens einer Exazerbation im zurückliegenden Jahr oder einer FEV1 unter $60 \%$ und keiner Exazerbation. Kontrollen waren Patienten ohne COPD oder mit einer milden Form der Erkrankung (FEV $1>60 \%$ und keine Exazerbation im zurückliegenden Jahr). Alle Teilnehmer füllten einen ausführlichen Fragebogen mit 44 Punkten aus, den die Autoren unter anderem anhand früherer Untersuchungen und aktueller Literatur zusammengestellt hatten. Mittels einer Random-Forest-Analyse ermittelten die Autoren die kleinste Auswahl an Fragen, die in Kombination mit einer Peak-Flow (PEF)-Messung Patienten mit größtmöglicher Sensitivität und Spezifität identifizieren konnte.
186 Fälle und 160 Kontrollen mit PEFMessungen gingen in die Analyse ein. Das Durchschnittsalter der Gesamtkohorte lag bei 62,7 Jahren, 55\% von ihnen waren weiblich und $16 \%$ hatten niemals geraucht. Das mittlere FEV1 betrug bei den Fällen $42,5 \%$ und bei den Kontrollen 82,5\%. Aus dem Fragenkatalog entwickelten die Autoren den 5Punkte-Fragebogen CAPTURE (SOPD Assessment in Primary Care to identify Undiagnosed Respiratory Disease and Exacerbation Risk). Dieser beinhaltete die Bereiche Exposition gegenüber Noxen, Atemprobleme, Belastungsdyspnoe, Ermüdbarkeit und Arbeitsausfälle aufgrund respiratorischer Probleme. Die Kombination aus Fragebogen und PEFMessung (<350l/min für Männer und $<250$ l/min für Frauen) konnte mit einer Sensitivität von $89,7 \%$ und einer Spezifität von 78,1\% Fälle von der Gesamtkohorte der Kontrollen unterscheiden sowie mit einer Sensitivität von 89,7\% und einer Spezifität von 93,1\% Fälle von Nicht-COPD-Kontrollen.

\section{FAZIT}

Der aus fünf einfachen Fragen bestehende Fragebogen CAPTURE in Kombination mit einer Peak-Flow-Messung konnte nach Ansicht der Autoren Patienten mit einer COPD identifizieren, die von den derzeit verfügbaren Therapien profitieren würden und bei denen weitere diagnostische Maßnahmen erforderlich sind. Dies könne vor allem im ambulanten Bereich hilfreich sein. Für die Zukunft seien weitere Studien notwendig, um die Leistungsfähigkeit dieser Methode in diesem Setting zu beurteilen.

Dr. med. Johannes Weiß, Bad Kissingen 